\title{
Dual-Energy Subtraction X-Ray Digital Tomosynthesis: Basic Physical Evaluation
}

\author{
Tsutomu Gomi ${ }^{1}$, Masahiro Nakajima ${ }^{2}$ \\ ${ }^{1}$ School of Allied Health Sciences, Kitasato University, Sagamihara, Japan \\ ${ }^{2}$ Department of Radiology, Dokkyo Medical University Hospital, Koshigaya, Japan \\ Email: gomi@kitasato-u.ac.jp
}

Received June 7, 2012; revised July 11, 2012; accepted July 25, 2012

\begin{abstract}
The present study was performed to determine the potential of applying dual-energy subtraction (DES) digital tomosynthesis to basic physical evaluations. Volumetric X-ray DES digital tomosysnthesis was used to obtain an image of a detectability phantom (an artificial lesion). The image quality of DES digital tomosynthesis was compared to that of DES radiography. The purpose of this study was to determine enhanced visibility quantified in terms of the contrastto-noise ratio, figure-of-merit, and root-mean-square error. In the in-focus plane, the image quality is better by DES digital tomosynthesis than by DES radiography. The potential usefulness of DES digital tomosynthesis for evaluating a detectability phantom was demonstrated. Further studies are required to determine the ability of DES digital tomosynthesis to quantify the spatial relationships between the artificial lesion components of these devices, as well as to identify lesions with diagnostic consequences.
\end{abstract}

Keywords: Digital Tomosynthesis; Dual-Energy Subtraction

\section{Introduction}

Dual-energy subtraction (DES) imaging can be used to improve the conspicuity of a specific tissue. With DES imaging, the image signal of one tissue material can be suppressed in order to remove anatomic background noise, thereby enhancing the contrast of the feature of interest. DES involves making two radiographic projections of the patient using X-ray beams of different energies. By exploiting the difference in the energy dependence of attenuation between bone and soft tissue, the contrast of the bone can be reduced to produce a softtissue-only image, and the contrast of the soft tissue can be reduced to produce a bone image [1]. Computed tomography (CT) scanners were used in the Scout-view mode [2], and an even film has been proposed as a dualenergy receptor [3]. Recent computed radiography (CR) systems have been hampered by poor subtraction effectiveness, workflow inconveniences, and detective quantum efficiency limitations in the CR technology. DES, in conjunction with a flat-panel detector (FPD), is now commercially available for chest radiography applications [4]. DES has been applied to the detection of calcium in pulmonary nodules $[5,6]$, bone mineral analysis [7], and cardiac imaging [8].

Although DES imaging increases object conspicuity and detectability by removing anatomic background noise, recent advances in digital radiography with FPDs

Copyright (C) 2012 SciRes. offer the possibility of increased DES performance. With their high quantum efficiency, efficient dose utilization, and fast-frame readout capabilities, new possibilities for DES imaging with FPDs exist for applications in both digital tomosynthesis and digital radiography.

Conventional tomography provides planar information about an object from its projection images. In tomography, an X-ray tube and an X-ray film receptor are placed on either side of the object. The relative motions of the tube and film are predetermined by the desired location of the in-focus plane. A single image plane is generated by a scan; however, multiple scans may be required to provide a sufficient number of planes to cover the selected structure in the object. In digital tomosynthesis imaging, only one set of discrete X-ray projections can be used to retrospectively reconstruct any plane of the object. This technique has been investigated for angiographic analysis, as well as for chest, hand joint, pulmonary, dental, and breast imaging analyses [9].

DES digital tomosynthesis is a new technique; therefore, there is little guidance for its integration into the clinical practice of radiography [10-12]. In this background, we focus on the possibility of detecting calcium in pulmonary nodules, specifically for chest diagnostics. The goal of this study is to investigate the effects of different DES techniques (digital tomosynthesis vs. digital radiography) and determine a combination that would 
optimize system performance given some clinically relevant experimental parameters.

\section{Materials and Methods}

\subsection{DES Algorithm}

We assumed monoenergetic X-ray beams denoted by $H$ and $L$ and an object made of two materials, bone and soft tissue $(B, S)$. The intensity of the transmitted X-ray is given by

$$
\begin{gathered}
I_{L}=I_{0 L} \cdot e^{-\left(\mu_{B L} \cdot x_{B}+\mu_{S L} \cdot x_{S}\right)} \\
I_{H}=I_{0 H} \cdot e^{-\left(\mu_{B H} \cdot x_{B}+\mu_{S H} \cdot x_{S}\right)}
\end{gathered}
$$

where $L$ and $H$ denote either the low- or high-energy Xrays, $I_{0}$ and $I$ are the incident and transmitted intensities of the X-ray beam, $\mu_{B}$ and $\mu_{S}$ are the linear attenuation coefficients of bone and soft tissue, and $x_{B}$ and $x_{S}$ are the thickness of bone and soft tissue in the patient, respectively.

By considering a statement logarithm on Equations (1) and (2), we arrive at

$$
\begin{aligned}
& \log \left(\frac{I_{H}}{I_{0 H}}\right)=-\mu_{B H} \times x_{B}-\mu_{S H} \times x_{S} \\
& \log \left(\frac{I_{L}}{I_{0 L}}\right)=-\mu_{B L} \times x_{B}-\mu_{S L} \times x_{S}
\end{aligned}
$$

Equations (3) and (4) can expressed as follows.

$$
\begin{aligned}
& \frac{\mu_{S L}}{\mu_{S H}} \log \left(\frac{I_{H}}{I_{0 H}}\right)-\log \left(\frac{I_{L}}{I_{0 L}}\right)=x_{B} \times\left(\mu_{B L}-\frac{\mu_{S L}}{\mu_{S H}} \mu_{B H}\right) \\
& \frac{\mu_{B L}}{\mu_{B H}} \log \left(\frac{I_{H}}{I_{0 H}}\right)-\log \left(\frac{I_{L}}{I_{0 L}}\right)=x_{S} \times\left(\mu_{S L}-\frac{\mu_{B L}}{\mu_{B H}} \mu_{S H}\right)
\end{aligned}
$$

where $\frac{\mu_{S L}}{\mu_{S H}}$ and $\frac{\mu_{B L}}{\mu_{B H}}$ are weighting factors, and Equation (5) represents a process to enhance soft tissues and Equation (6) to enhance bone.

\subsection{DES Digital Tomosynthesis and Des Digital Radiography Systems}

The DES digital tomosynthesis system (SonialVision Safire II, Shimadzu Co., Kyoto, Japan) consisted of an X-ray tube with a $0.4-\mathrm{mm}$ focal spot and a $432 \times 432$ $\mathrm{mm}$ amorphous selenium digital FPD with a detector element size of $150 \times 150 \mu \mathrm{m}$. The motion of the collimator was synchronized with the tube motion constantly measuring the misalignment of low-kVp and high-kVp images. The image intensifier of the conventional DES tomosynthesis system used tube voltages of 60 and 120 $\mathrm{kVp}$ in clinical [13] and phantom [12] trials of chest examinations, respectively. In the FPDs of DES digital to- mosynthesis imaging, pulsed X-ray exposures were used with rapid switching between low $(60 \mathrm{kVp})$ and high energies $(120 \mathrm{kVp})$. Tomography was performed with a linear tomographic movement of the system, a scan time of $6.4 \mathrm{~s}$, and a swing angle of $40^{\circ}$. Thirty-seven low- and high-voltage projection images were sampled during a single tomographic pass (Figure 1). These images were sampled at a matrix size of $2880 \times 2880$ by 12 bits and were used to reconstruct low- and high-voltage tomograms of any desired height. Bone or soft tissue tomograms were produced by weighted subtraction of each of the different absorption coefficients. Each projection image was acquired at $200 \mathrm{~mA}$ and a 20 - 25-ms exposure time for lowvoltage X-rays, and at $200 \mathrm{~mA}$ and a $\leq 25$-ms exposure time for high-voltage X-rays. The reconstitution slice thickness and reconstruction interval were fixed at $1 \mathrm{~mm}$. An antiscatter grid (focused type, grid ratio 12:1) was used. The DES digital tomosynthesis images used filtered backprojection in their reconstruction [14].

The DES radiography system (SonialVision Safire II, Shimadzu Co., Kyoto, Japan) consisted of an X-ray tube with a $0.4-\mathrm{mm}$ focal spot and a $432 \times 432-\mathrm{mm}$ amorphous selenium digital FPD with a detector element size of 150 $\times 150 \mu \mathrm{m}$. DES radiography images were processed from low- and high-voltage projection images by two rounds of double-exposure acquisition (Figure 1). Images were sampled at a matrix size of $2880 \times 2880$ by 12 bits and were used to reconstruct low- and high-voltage tomograms at any desired height. Bone or soft tissue images were produced by weighted subtraction of each of the different absorption coefficients. Each projection image was acquired at $200 \mathrm{~mA}$ and a $20-25-\mathrm{ms}$ exposure time

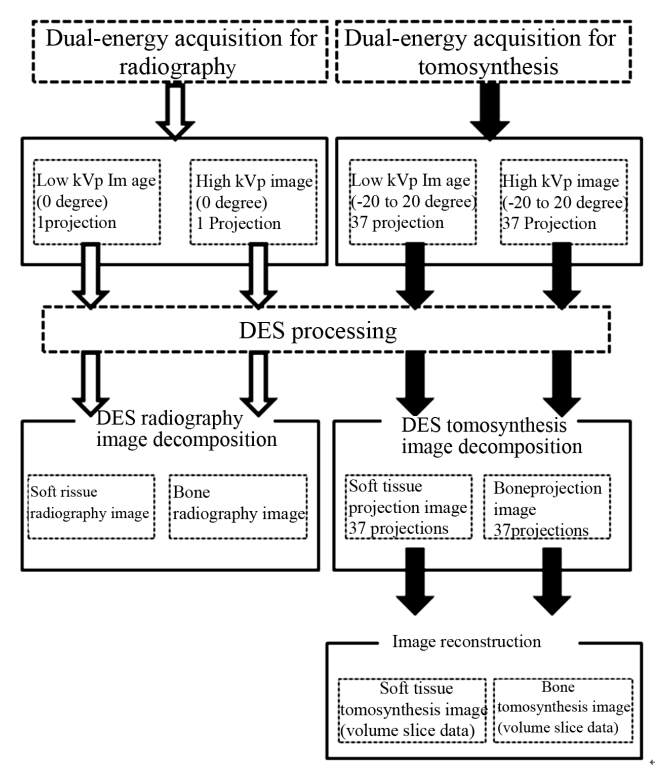

Figure 1. Illustration of the imaging sequence by dual-energy subtraction digital tomosynthesis imaging and dualenergy subtraction radiography. 
for low-voltage X-rays and at $200 \mathrm{~mA}$ and a $\leq 25$-ms exposure time for high-voltage X-rays. An antiscatter grid was used (focused type, grid ratio 12:1).

\subsection{Phantom Specification}

For image quality evaluations, the radiation to the detectability phantoms was attenuated using polyurethane slabs. Detectability phantoms (artificial lesion region; $\mathrm{CaCO}_{3}$ ) of different diameters and thicknesses were arranged within the polyurethane slabs (Figure 2). Radiograms were obtained using a detectability phantom, while the evaluation object was used for bone imaging. Polymethyl methacrylate (PMMA) steps (1 cm in size) were imaged over the PMMA stack whose thicknesses ranged between 5,10 , and $15 \mathrm{~cm}$.

\subsection{Evaluation Methods}

The detectability phantom was used to compare image performance of DES digital tomosynthesis and DES digital radiography. DES processing techniques such as contrast-to-noise ratio (CNR), figure-of-merit (FOM), root-mean-square error (RMSE) were evaluated. In the DES digital tomosynthesis image evaluation, different reconstruction kernels [14] of the three components (cutoff frequencies $0.04,0.02$, and 0.01 cycles $/ \mathrm{mm}$ ) were compared in the in-focus plane. The evaluation item was established in four marks (large1, large2, small1, small2; Figure 2). In this study, the evaluation object was used for a bone image.

\subsubsection{Contrast-to-Noise Ratio (CNR)}

CNR per pixel was calculated as a measure of detectability of $\mathrm{CaCO}_{3}$ in the images. CNR was defined as

$$
C N R=\frac{S_{\text {Background }}-S_{\text {Object }}}{\sigma_{\text {Backgound }}}
$$

A square region of interest (ROI) was drawn on either the image background or the object in the image in order to measure the signal level. The noise was taken as the standard deviation in the background ROI; $S_{\text {Object }}$ is the mean pixel value in ROI within the object, $S_{\text {Background }}$ is the mean pixel value in the background ROI, and $\sigma_{\text {Backgound }}$ is the standard deviation of pixel values in the background ROI (Figure 2). Throughout these results, $\sigma_{\text {Backgound }}$ included structure noise that can obscure the object, not just photon statistics and electronic noise.

\subsubsection{Figure-of-Merit (FOM)}

FOM [15] was defined as

$$
F O M=\frac{C N R^{2}}{X^{L}+X^{H}}
$$

where $X^{L}$ and $X^{H}$ are the low- and high-energy expo-

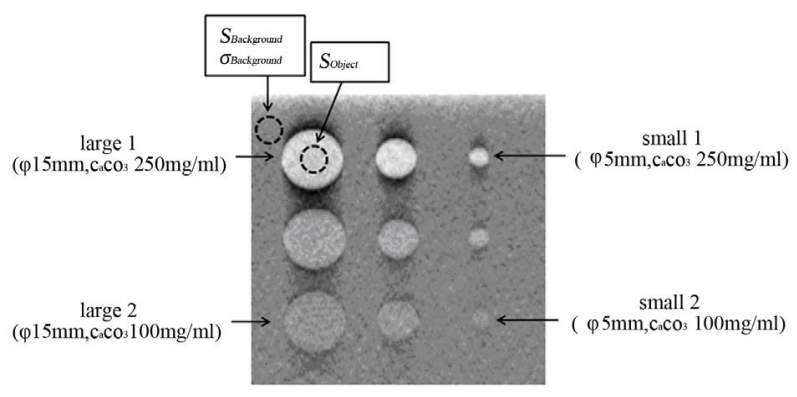

Figure 2. X-ray exposure acquisition setting for a single projection of the detectability phantom Areas of measurement for the evaluation and four analysis object metrics (large 1, large 2, small 1, and small 2) are shown.

sures, respectively. FOM includes both measures of image quality and patient risk. It provides a single value measure of system performance and allows for an easy comparison between systems and different configurations of the same system. To establish the entrance surface dose, a glass dosimeter was used to measure the X-ray tube output.

\subsubsection{Root-Mean-Square Error (RMSE)}

The performance of the DES digital tomosynthesis and DES digital radiography systems was compared by evaluating the RMSE of each image (no PMMA images of any size, i.e., 5,10 , or $15 \mathrm{~cm}$, were taken).

RMSE was defined as

$$
\operatorname{RMSE}=\sqrt{\frac{\sum_{i=1}^{n}\left(\hat{y}_{k}-y_{k}\right)^{2}}{n}}
$$

where $y_{k}$ is the observed image, $\hat{y}_{k}$ is the referenced image (no PMMA image), and $n$ is the number of compounds in the analyzed set.

\section{Results}

Processing of the high-contrast detectability phantom gave clear contrast detectability by DES digital tomosynthesis imaging, and DES digital radiography produced an increase in the number of same images (Figure 3). Regarding the images of similar signal size and $\mathrm{CaCO}_{3}$ status, images processed by DES digital tomosynthesis had greater contrast than those processed by DES digital radiography.

The CNR, FOM, and the quality of images produced by DES digital tomosynthesis were significantly superior to those obtained by DES digital radiography for DES digital tomosynthesis imaging. The results confirm that the CNR value increases when viewing a small signal (small1, small2) in the presence of $\mathrm{CaCO}_{3}$ (Figures 4 and 5).

The RMSE and error images produced by DES digital radiography were significantly superior to those obtained 
by DES digital tomosynthesis for DES digital radiography imaging (Figure 6).

\section{Discussion}

DES imaging has been proposed and investigated by many researchers as a means of reducing the impact of anatomic "noise" on disease detection by radiography.
DES involves making two radiographic projections of the object using different energy X-ray beams. By exploiting the difference in the energy dependence of attenuation between the bone and soft tissue, the bone contrast can be reduced to produce a soft tissue image, and the contrast of the soft tissue can be reduced to produce a bone image. Conventional digital radiography systems have

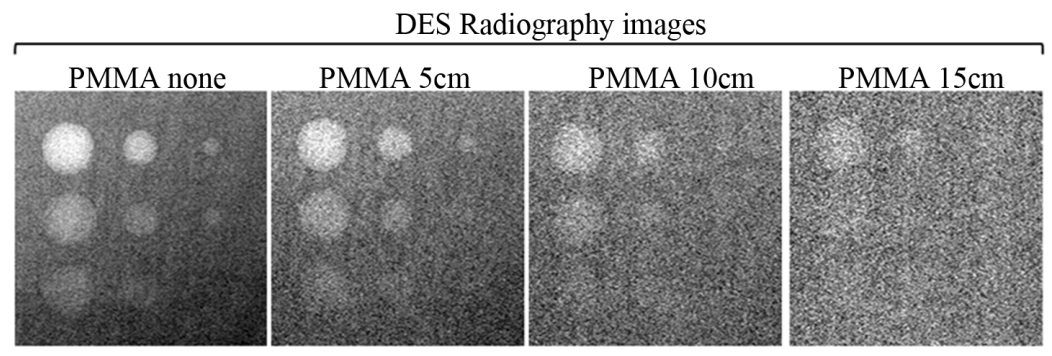

DES Tomosynthesis images(cut-off frequency;0.01 cycles/mm)

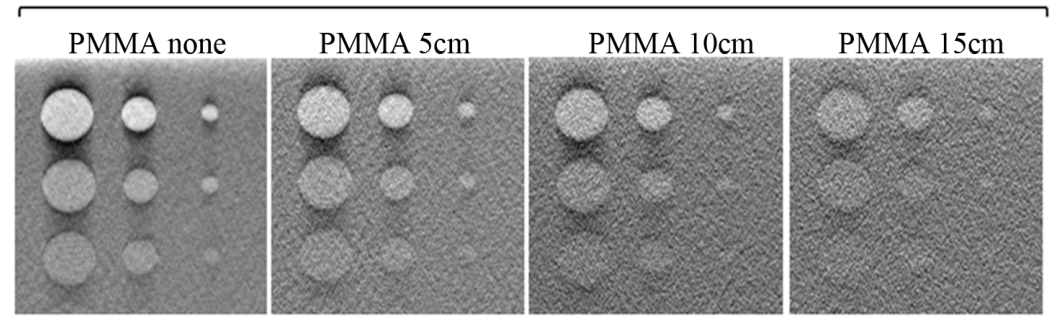

Figure 3. Comparison of detectability phantom image (bone image) with images obtained from dual-energy subtraction digital tomosynthesis (cut-off frequency, $0.01 \mathrm{cycles} / \mathrm{mm}$ ) of the in-focus plane, and dual-energy subtraction radiography. Dualenergy subtraction digital tomosynthesis provided better visualization of the detectability contrast.

large 1

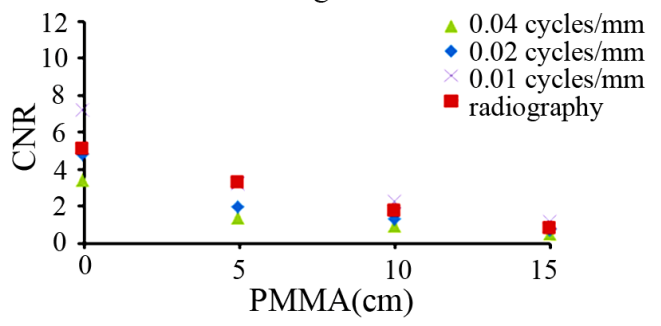

small 1

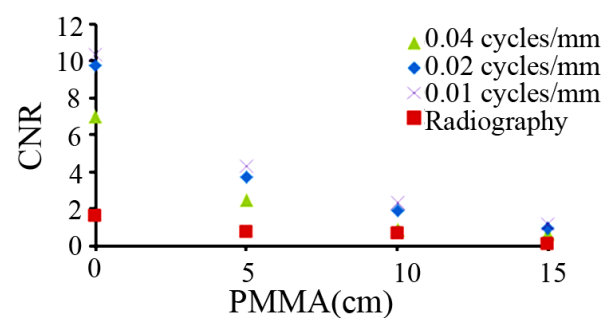

large 2

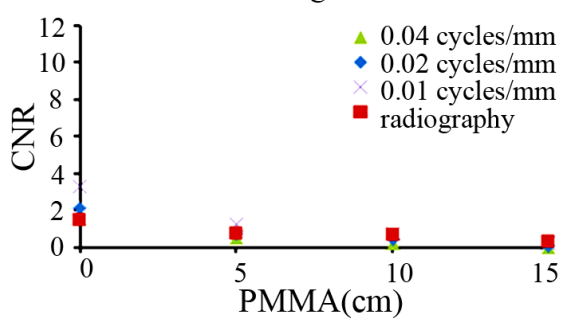

small 2

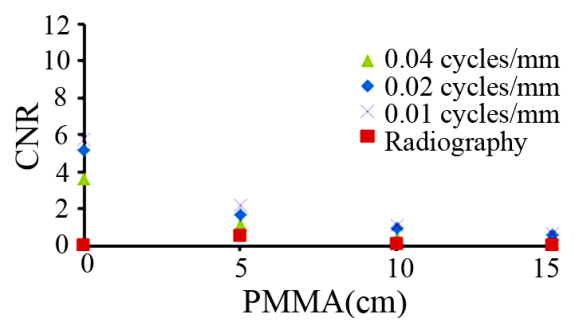

Figure 4. Effect of a dual-energy subtraction tomosynthesis of different reconstruction kernels and dual-energy subtraction digital radiography on CNR. A dual-energy subtraction digital tomosynthesis can confirm that the CNR value increases without direct exposure to large-size $\mathrm{CaCO}_{3}$ content. 
large 1

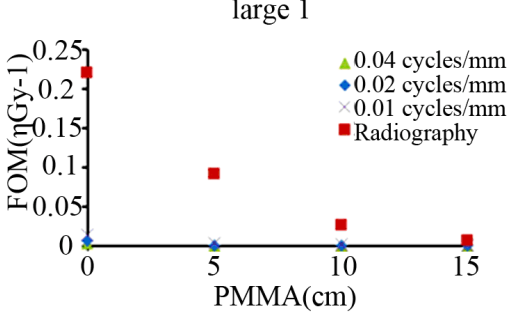

small 1

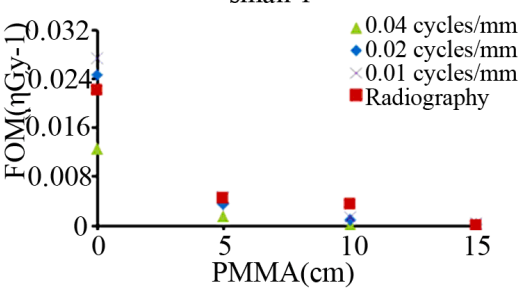

large 2

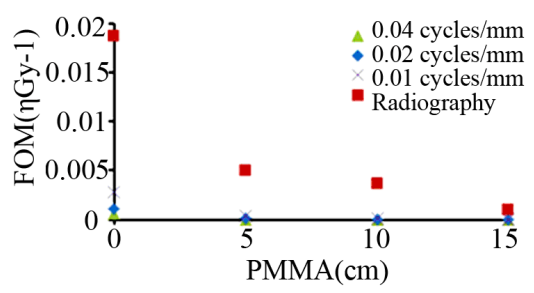

small 2

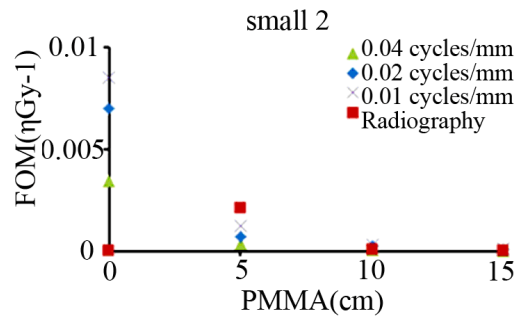

Figure 5. Effect of a dual-energy subtraction tomosynthesis of different reconstruction kernels and dual-energy subtraction digital radiography on FOM. A dual-energy subtraction digital tomosynthesis can confirm that the FOM value increases without direct exposure to large-size $\mathrm{CaCO}_{3}$ content.
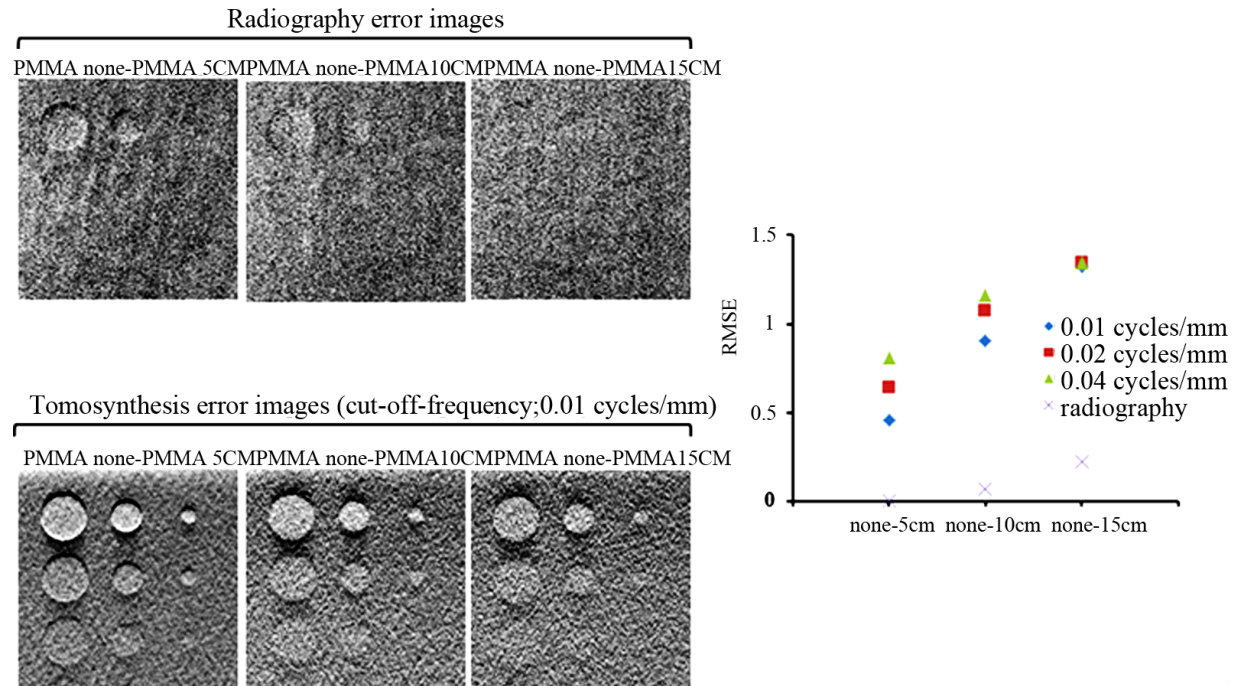

Figure 6. Comparison of RMSE with error images obtained from dual-energy subtraction digital tomosynthesis of different reconstruction kernels and the in-focus plane and dual-energy subtraction digital radiography.

been hampered by poor subtraction effectiveness, workflow inconveniences, and limitations in detective quantum efficiency of the technology. Projection images acquired with DES techniques, however, are susceptible to overlap of anatomic features [16-19].

Quantum noise plays an important role in the degradation of contrast resolution of radiographs. It increases inversely with X-ray exposure and constitutes the dominant noise source at low-radiation exposure levels. Because of quantum noise, the technical factors used to reduce the radiation dose in the DES digital tomosynthesis system are limited to those levels usually employed in conven- tional digital tomosynthesis. However, synthesized tomograms can be obtained with the same technical specifications used for digital radiography when the presence of quantum noise can be tolerated. The information contained in a DES digital tomosynthesis voxel is obtained from 74 projections. Ideally, the number of X-ray quanta for the reconstruction of this voxel equals that from a single projection acquired with the same amount of total exposure. Considering the detector noise from the acquisition of each projection, CNR is reduced in radiography imaging, which distributes the total exposure over one projection. 
In conventional tomosynthesis imaging, the type of motion used during data acquisition dictates the type of blurring of off-focal-plane objects in the image. On the other hand, three-dimensional reconstruction schemes such as digital tomosynthesis and $\mathrm{CT}$ require complete knowledge of the X-ray source projection geometry prior to exposure. This limitation precludes much of the potential task-dependent flexibility. This limitation also precludes accurate reconstruction from projections acquired from an object that moves unpredictably between exposures, as this is equivalent to not knowing the projection geometry. However, blurring cannot be eliminated by DES; it will still lead to inaccurate reconstruction from projections. DT overcomes these difficulties by enabling reconstruction of numerous image slices from a single low-dose image data acquisition. DT images are invariably affected by blurring due to objects lying outside the plane of interest and superimposed on the focused image of the fulcrum plane by limited acquisition angle. This results in poor object detectability in the in-focus plane.

Initial data from our study suggest that DES digital tomosynthesis will substantially enhance sensitivity and specificity of the detectability phantom study. Gomi et al., presents a comparative study of chest DES digital tomosynthesis and DES radiography with respect to their efficacy for detecting simulated pulmonary nodules with and without calcifications. The statistical evaluation results indicated that digital tomosynthesis performed better in detecting nodules than DES [12]. Carton et al., study is to assess the feasibility of DES as a technique for contrast-enhanced digital tomosynthesis. Contrast-enhanced DES digital tomosynthesis was able to provide morphorogy and kinetic information about the known malignancy [10]. Despite its potential, DES digital tomosynthesis is a new technique. In addition, this technique is not susceptible to the problems of image overlap, partial volume effect, or shifting of the image plane.

\section{Conclusion}

We conclude that DES digital tomosynthesis is superior to DES digital radiography for detecting phantom objects with artificial lesion regions. We believe that DES digital tomosynthesis imaging quality can improve the detection of lesion regions when applied in clinical practice.

\section{REFERENCES}

[1] W. R. Brody, G. Butt, A. Hall and A. Macovski, "A Method for Selective Tissue and Bone Visualization Using Dual-Energy Scanned Projection Radiography," Medical Physics, Vol. 8, No. 3, 1981, pp. 353-357. doi:10.1118/1.594957

[2] L. A. Lehmann, R. E. Alvarez, A. Macovski, W. R. Brody, N. J. Pelc, S. J. Riederer and A. L. Hall, "Gener- alized Image Combinations in Dual KVP Digital Radiography," Medical Physics, Vol. 8, No. 5, 1981, pp. 659-667. doi: $10.1118 / 1.595025$

[3] R. A. Kruger, J. D. Armstrong, J. A. Sorenson and L. T. Niklason, "Dual Energy Film Subtraction Technique for Detecting Calcification in Solitary Pulmonary Nodules," Radiology, Vol. 140, No. 1, 1981, pp. 213-219.

[4] J. M. Sabol, G. B. Avinash, F. Nicolas, B. Claus, Z. Jianguo and J. T. Dobbins, "The Development and Characterization of a Dual-Energy Subtraction Imaging System for Chest Radiography Based On CsI:Tl Amorphous Silicon Flat-Panel Technology," Proceedings of SPIE on Physics of Medical Imaging, Vol. 4320, 2001, pp. 399408. doi:10.1117/12.430897

[5] R. G. Fraser, N. M. Hickey, L. T. Niklason, E. A. Sabbagh, R. F. Luna, C. B. Alexander, C. A. Robinson, A. L. Kat- zenstein and G. T. Barnes, "Caccification in Pulmonary Nodules: Detection with Dual-Energy Digital Radiography," Radiology, Vol.160, No. 3, 1986, pp. 595-601.

[6] L. T. Niklason, N. M. Hickey, D. P. Chakraborty, E. A. Sabbagh, M. V. Yester, R. G. Fraser and G. T. Brans, "Simulated Pulmonary Nodules: Detection with Dual-Energy versus Conventional Radiography," Radiology, Vol. 160, No. 3, 1986, pp. 589-593.

[7] R. A. Kuger, "Dual-Energy Electric Sanning-Slit Fluorography for the Determination of Vertebral Bone Mineral Content," Medical Physics, Vol. 14, No. 4, 1987, pp. 562 566. doi:10.1118/1.596068

[8] S. Molloi, A. Ersahin, J. Tang, J. Hicks and C. Y. Leung, "Quantification of Volumetric Coronary Blood Flow with Dual-Energy Digital Subtraction Angiography," Circulation, Vol. 93, No. 10, 1996, pp. 1919-1927. doi:10.1161/01.CIR.93.10.1919

[9] J. T. Dobbins and D. J. Godfrey, "Digital X-Ray Tomosynthesis: Current State of the Art and Clinical Potential," Physics in Medicine and Biology, Vol. 48, No. 19, 2003, pp. R65-R106. doi:10.1088/0031-9155/48/19/R01

[10] A.-K. Carton, S. C. Gavenonis, J. A. Currivan, E. F. Conant, M. D. Schnall and A. D. A. Maidment, "Dual-Energy Contrast-Enhanced Digital Breast Tomosynthesis: A Feasibility Study," British Journal of Radiology, Vol. 83, No. 988, 2009, pp. 344-350. doi:10.1259/bjr/80279516

[11] E. Samei and R. S. Saunders Jr., "Dual-Energy ContrastEnhanced Breast Tomosynthesis: Optimization of Beam Quality for Dose and Image Quality," Physics in Medicine and Biology, Vol. 56, No. 19, 2011, pp. 6359-6378. doi:10.1088/0031-9155/56/19/013

[12] T. Gomi, M. Nakajima, H. Fujiwara and T. Umeda, "Com- parison of Chest Dual-Energy Subtraction Digital Tomosynthesis Imaging and Dual-Energy Subtraction Radiog- raphy to Detect Simulated Pulmonary Nodules with and without Calcifications: A Phantom Study," Academic Ra-diology, Vol. 18, No. 2, 2011, pp. 191-196. doi:10.1016/j.acra.2010.09.021

[13] S. Sone, T. Kasuga, F. Sakai, J. Aoki, I. Izuno, Y. Tanizaki, H. Shigeta and K. Shibata, "Development of a HighResolution Digital Tomosynthesis System and Its Clinical Application," Radiographics, Vol. 11, No. 5, 1991, pp. 
807-822.

[14] T. Gomi and H. Hirano, "Clinical Potential of Digital Linear Tomosynthesis Imaging of Total Joint Arthroplasty," Journal of Digital Imaging, Vol. 21, No. 3, 2008, pp. 312-322. doi:10.1007/s10278-007-9040-9

[15] L. D. Justin, X. Tong and M. Sabee, "Dual-Energy Cardiac Imaging: An Image Quality and Dose Comparison for flat-Panel Detector and X-Ray Image Intensifier," Physics in Medicine and Biology, Vol. 52, No. 1, 2007, pp. 183-186. doi:10.1088/0031-9155/52/1/012

[16] N. M. Hickey, L. T. Niklason, E. Sabbagh, R. G. Fraser and G. T. Barnes, "Dual-Energy Digital Radiographic Quantification of Calcium in Simulated Pulmonary Nodules," American Journal of Roentgenology, Vol. 148, No.
1, 1987, pp. 19-24.

[17] T. Ishigaki, S. Sakuma, Y. Horikawa, M. Ikeda and H. Ya- maguchi, "One-Shot Dual-Energy Subtraction Imaging," Radiology, Vol. 161, No. 1, 1986, pp. 271-273.

[18] T. Ishigaki, S. Sakuma and M. Ikeda, "One-Shot DualEnergy Subtraction Chest Imaging with Computed Radiography: Clinical Evaluation of Film Images," Radiology, Vol. 168, No. 1, 1988, pp. 67-72.

[19] H. Nishitani, Y. Umezu, K. Ogawa, H. Yuzurihara, H. Tanaka and K. Matsuura, "Dual-Energy Projection Radiography Using Condenser X-Ray Generator and Digital Radiography Apparatus," Radiology, Vol. 161, No. 2, 1986, pp. 533-535. 